\title{
La gobernabilidad fluvial y sus dificultades en Colombia
}

\author{
River governance and its difficulties in colombia
}

\section{Luis Eduardo Chávez Perdomo}

Oficial Superior de la reserva activa (RA) de la Armada de Colombia, Máster en Derecho Internacional y relaciones Exteriores e Internacionales, Abogado Marítimo, Profesional en Ciencias Navales, Perito Naval, Profesor investigador, Becario en Gestión de Puertos CIP-OEA, Consultor, Miembro del Instituto Iberoamericano de Derecho Marítimo (IIDM)Rama Colombia y ACOLDEMAR Asociación Colombiana de Derecho Marítimo. lechp8@gmail.com

Fecha de recepción: marzo de 2020

Fecha de aprobación: julio de 2020

Para citar este artículo / To reference this article La gobernabilidad fluvial y sus dificultades en Colombia. Inciso, 22(1) ; 144-160.

DOI: http://dx.doi.org/10.18634/incj.22v.1i.1029

\section{Resumen}

El escenario de la gobernanza fluvial en Colombia encierra diversos desafíos administrativos que inciden en la protección y adecuado uso de los recursos naturales del país, por esta razón se propone como objetivo, mostrar cómo las divisiones jurisdiccionales del Estado que dificultan la administración del rio e identificar el o los responsables administrativos para el cuidado de este bien de uso público.

Para tal fin, este artículo desarrolla un análisis enfocado en la legislación, doctrina y políticas existentes en relación con este campo. Se busca entonces hacer una revisión sistemática legal, en las diferentes áreas, y buscando si lo hay una revisión secundaria, que se desarrolla en el tiempo donde toda la normativa se concreta en este caso en las orillas del rio, trabajo que se realiza en un semestre dada la complejidad por la cantidad de actividades que se pueden realizar. 
Se observó la existencia en diversidad y número de entidades a cargo, generando una dispersión en la gestión armónica y sinérgica sobre la administración de los ríos, al no ser visto como una sola unidad, sino que se encuentra administrado de forma fraccionada en cada una de las regiones que baña, o los servicios que pueda prestar, siendo complejo su manejo y cuidado. Es de urgencia reconocer la necesidad de generar cambios institucionales, políticas y lineamientos a largo plazo que permitan realizar una debida gestión de los recursos fluviales sin afectar el ecosistema en el cual se encuentra inmerso.

Palabras clave: Río, Cuenca, Administración de justicia, recursos naturales, política ambiental, desarrollo sostenible, legislación, autosuficiencia, responsabilidad.

\section{Abstract}

The scenario of river governance in Colombia encompasses various administrative challenges that affect the protection and proper use of the country's natural resources. It is urgent to identify institutional reforms that can address the proper management of river resources without affecting the ecosystem in which it is immersed. To this end, this article develops an analytical methodology to study the current legislation, and the design of implement plans and projects of the State in relation to this field. It will be observed there are many units in charge; the result is the river is not seen as a unit, but it is divided and in real terms managed with different guidelines for each of the regions that it bathes.

Keywords: río, Cuenca, Administración de justicia, recursos naturales, política ambiental, desarrollo sostenible, legislación, autosuficiencia, responsabilidad.

\section{Introducción}

La gobernabilidad, refiere a la implementación efectiva y socialmenteaceptada de responsabilidad y regulación y es, por lo tanto, de una naturaleza extremadamente política. (Hall P. R., 2003) La gobernanza es un concepto más inclusivo que el de gobierno por sí solo, ya que abarca la relación entre una sociedad y su gobierno. Por lo general involucra un comportamiento de mediación a través de valores, normas y, donde sea posible, mediante leyes (Hall, 2003, pág. 3).

El tema de la gobernanza del rio y sus recursos genera gran debate en cualquier país, debido a la cantidad de actividades y usos que se le dan y a las de autoridades que cada Estado propone para explotarlo y controlar el recurso. En Colombia, la administración pública se ha dividido en entidades territoriales, como lo son las regiones, departamentos o municipios. Ellos, están bañados por algún cuerpo de agua y sostienen actividades dependientes del recurso hídrico como el transporte, la pesca, la energía, el uso doméstico o el riego. Como se observará, el gobierno ha fraccionado la legislación y el ordenamiento del rio por temas específicos (medio ambiente, proyección urbanística, transporte entre otros) los cuales muchas veces se encuentran distanciados entre sí, lo que provoca que no se tenga en cuenta las características y los sistemas 
generales propios de las cuencas, como contextura natural, esta situación a pesar de que la norma establecida busca su cuidado no está alineada ni tiene procedimientos o controles para cumplirlo, con el fin de entender la complejidad del ente natural y la alineación en temas de política pública pues una acción aguas arriba traerá necesariamente consecuencias aguas abajo.

En este orden ideas, el objetivo principal de este artículo es mostrar cómo las divisiones jurisdiccionales del Estado dificultan la administración del recurso hídrico. Pese a la relevancia del tema, las investigaciones realizadas en materia de gobernabilidad y políticas de recursos hídricos se dirigen principalmente hacia la gestión de los mares y costas dejando de lado la importancia geopolítica, ambiental y económica que brindan los ríos y sus cuencas.

El presente trabajo, se desarrolló a través de la revisión primaria y secundaria de fuentes bibliográficas, el contexto jurídico durante un periodo de un semestre. Es una investigación realizada a título personal tras la observación de años de experiencia en el desarrollo de trabajos en materias de transporte fluvial e hidro estrategia y es desarrollado bajo los parámetros de una investigación legal básica de tipo descriptivo y con alcance exploratorio partiendo de estudios realizados por el mismo gobierno y del análisis de fuentes bibliográficas enfocadas en el estudio las fuentes hídricas en Colombia.

\section{Marco general}

El termino gobernanza representa la construcción de canales de comunicación entre gobernantes y gobernados. Tiene implícita la formación de iniciativas, acuerdos y alianzas que conduzcan al bienestar colectivo. La gobernanza de los recursos hídricos implica la prestación y la garantía de los derechos del agua, su compleja administración y la preservación del conjunto aledaño de bienes que corren a lo largo del curso del rio.

La gobernanza fluvial no se puede enfocar únicamente en la administración y abastecimiento del recurso hídrico, se debe ampliar a todos los elementos que lo componen, que son parte de su naturaleza, así, por ejemplo, en los ríos debe ampliarse al conocimiento de sus riberas, sobre su cuenca con todas sus peculiaridades. Esta última incluso ve por la vegetación, su riqueza forestal debe cuidar los bordes del río para evitar inundaciones e incluso su riqueza animal, piscícola, entre otras, las cuales intervienen y permiten la protección del sistema fluvial. Estas características impactan directamente en la forma como debe ser gobernada.

Por esta razón, la administración de la cuenca permite la elaboración de políticas y acuerdos alineados a partir de una agenda pública, nacional e incluso internacional, así como de iniciativas que impactarán la región donde se encuentra la cuenca y sus habitantes y se buscará el aprovechamiento de los recursos para permitir un desarrollo sostenible.

La gobernanza fluvial, es un sistema de gestión política, que permite iniciativas que inciden en la inclusión social de toda una región bañada por un recurso hídrico de carácter fluvial determinado. Lo anterior hace veraz el dominio del Estado, sobre la utilización y uso del recurso. Así las cosas, es necesaria la fijación de estrategias de optimización combinadas con saberes de conservación, explotación, y manejo sustentable que conduzcan a una eficiente utilización que 
reúna a todos los actores para que puedan utilizar el recurso partiendo de que todo es posible si se conoce la capacidad cierta de la cuenca.

Como lo indica García (2015),

“la administración de los recursos hídricos supone un sistema sociopolítico de propensión a la sustentabilidad en el que la diseminación del poder político y la influencia social está delimitada por las oportunidades, capacidades y responsabilidades de los agentes económicos, actores, políticos y sujetos sociales" (García et al., 2015, pág.149).

Cuando el sistema de gobierno no cumple estas condiciones se puede decir que hay una gobernanza deficiente. Esta atmosfera conlleva a la generación de inestabilidad política, social y estructural del Estado y, más grave aún a la incapacidad de solucionar problemas compartidos (Royers \& Hall, 2003). La gobernanza debe estar alineada de tal forma que no existan zonas grises que permitan que la autoridad sea eficiente en su mandato. Muchas autoridades pueden facilitar el uso del recurso de manera indiscriminada y el ejercicio de la competencia puede verse visto afectado por la falta de medios reales que permitan la verificación de su control. Un ejemplo de ello es la desaparición del río Sambingo que hace parte de la cuenca hidrológica del Pacífico caucano, afectando la seguridad hídrica del país (Revista Semana, 2016).

En este sentido, la gobernabilidad del agua se refiere a la gama de sistemas políticos, sociales, ambientales, económicos y administrativos que existen para regular el desarrollo y la gestión de los recursos hídricos y el suministro de servicios asociados al agua.

\section{Marco regulador}

Colombia, tiene una gran cantidad de normas que tratan temas específicos sobre la gestión hídrica del agua como recurso, fija diferentes competencias de gobernanza no solo del recurso si no de su cuenca. Las normas que comprometen esta materia se encuentran dispersas en varios contenidos legales y reglamentarios que lo regularizan. Este artículo muestra de forma crítica el marco legal general que rodea el tema de los recursos hídricos, con especial atención al manejo de ríos, riberas y cuencas y sus problemáticas.

\section{La Constitución Política}

El tema ambiental tiene preceptos constitucionales, por lo que muchos califican la carta magna como ecológica. El artículo 79 de la constitución política señala:

“Todas las personas tienen derecho a gozar de un ambiente sano. La ley garantizará la participación de la comunidad en las decisiones que puedan afectarlo. Es deber del Estado proteger la diversidad e integridad del ambiente, conservar las áreas de especial importancia ecológica y fomentar la educación para el logro de estos fines" (Constitución Política, 1991).

Por lo tanto, la constitución vela no solo por el derecho a un medio ambiente sano, sino también por la inclusión de la sociedad en temas ambientales la cual, redunda en un mayor ejercicio de la gobernabilidad en temas ambientales. Esto es contundente, pues ayuda a entender la problemática ambiental cuidando las zonas de uso público que tienen los ríos dentro. 


\section{El Código Civil Colombiano}

En referencia a los bienes de uso público, el código civil, preceptúa sobre la propiedad de las aguas, en el artículo 677, establece que:

"Los ríos y todas las aguas que corren por cauces naturales son bienes de la Unión, de uso público en los respectivos territorios" y agrega que "Exceptúense las vertientes que nacen y mueren dentro de una misma heredad: su propiedad, uso y goce pertenecen a los dueños de las riberas, y pasan con estos a los herederos y demás sucesores de los dueños".

Lo anterior, explica sencillamente que es el Estado el que tiene la potestad de administrar las aguas y da un alcance claro sobre el uso de los bienes de uso público a toda la población en general, eso sí, con los lineamientos legales que le sean pertinentes y determina ciertas reglas que limitan su uso y clarifica la excepción para el curso de agua que nace y muere en una propiedad.

\section{En materia ambiental}

El plan nacional de desarrollo 2018- 2022 (DNP, 2019) direcciona los compromisos y metas del gobierno. Está compuesto por tres pactos estructurales, trece transversales y nueve por la productividad y la equidad en las regiones. Aunque dentro del pacto por la sostenibilidad se proyecta el cuidado y la conservación de los recursos naturales el tema del agua aparece de forma paralela dentro de otros pactos. Lo anterior implica que para alcanzar un "pacto por los recursos hídricos" es necesaria la comunicación e interacción entre las distintas entidades estatales y los sectores productivos que se encuentran vinculados al proceso.

El plan de desarrollo contempla: "conservar la conectividad ecosistémica a través de la formulación de los Planes de Ordenación y Manejo de Cuencas Hidrográficas (POMCAS)” (pág.1114). Igualmente, los pactos regionales reconocen dentro de sus objetivos de sostenibilidad: "Ia preservación y restauración de cuencas hidrográficas que alimentan las fuentes hídricas del país" (pág.1207). Todo ello, con el fin de alinearse con los objetivos de desarrollo sostenible ODS impulsados por la Organización de las Naciones Unidas (ONU).

Para el manejo de cuencas compartidas existe el deber constitucional del Estado de cooperar con otras naciones en la protección de ecosistemas fronterizos (art. 80 C.P.) además, establece que el Estado debe promover la internacionalización de las relaciones políticas, económicas, sociales y ecológicas sobre bases de equidad, reciprocidad y conveniencia nacional (art. 226 C.P.).

La posibilidad de adelantar programas de integración y cooperación fronteriza en temas ambientales está prevista expresamente en el artículo 289 de la Carta, que otorga facultades a las entidades territoriales limítrofes para que en forma directa puedan adelantar programas de preservación ambiental con las entidades vecinas de su misma categoría, con lo cual se determinó una excepción a la función de dirigir las relaciones internacionales del país, que tradicionalmente está bajo la tutela del Presidente de la República, lo que no ha tenido hasta la fecha desarrollos prácticos y no ha buscado facilitar el manejo de la cuenca como sistema de alta complejidad. 
Colombia se ha suscrito a diversos acuerdos multilaterales y bilaterales que promueven la protección medioambiental. Ejemplos de ellos son: el acta de San Pedro Alejandrino, de marzo 6 de 1990, entre Venezuela y Colombia y Acuerdo de Integración Subregional Andino en el que participan Bolivia, Colombia, Ecuador y Perú; ambos acuerdos proponen el logro del uso racional de los recursos hídricos, de esta forma se articula el Plan de Desarrollo que ordena integrar en una ley marco y en una sola política la normatividad y la política existente. (Iza, 2006).

A nivel interno Colombia cuenta con las Corporaciones Autónomas Regionales (CAR) las cuales diseñan políticas, planes y proyectos medioambientales, así como efectúan el cumplimiento de las disposiciones expedidas por el ministerio de ambiente (Ley 99 de 1993, artículo 31).

Aunque la ley establece que las distintas CAR del país se definirán por las características que constituyen geográficamente un mismo ecosistema, muchas veces estas entidades responden más a los límites político- administrativos. En ocasiones no coinciden con criterios técnicos, ni ecológicos, ni sistémicos y tampoco tienen en cuenta las cuencas que dan muchos de los nombres a estas entidades ambientales, convirtiéndose en una de las grandes vulnerabilidades de la administración.

\section{Los POT (planes de ordenamiento territorial)}

Los Planes de ordenamiento territorial (POT) son los instrumentos básicos establecidos por la ley utilizados por los municipios para reglamentar el uso del suelo (ley 388 de 1997), que aplica a todos los municipios ribereños y en ellos está la responsabilidad de su cuidado y uso.

La inclusión de la gobernanza fluvial dentro de los planes de ordenamiento territorial, en los planes de desarrollo municipal o regional debe ser vista como la mirada a las oportunidades económicas. Es común ver como el rio, sus zonas aledañas y su cuenca se miran con frecuencia como los botaderos de desperdicios y vertederos de residuos, esa imagen devastadora que simplemente recuerda cómo la contaminación afecta al hombre, su salud y las actividades para su desarrollo; sin embargo, al lograr incluir la cuenca en el desarrollo municipal, se adquieren espacios de invaluable valor paisajístico con efectos importantes en la economía local y produciendo una identidad propia a sus habitantes, ejemplos recientes son los malecones de Barranquilla o Buenaventura, inspirados en Puerto madero en Argentina, o Guayaquil en Ecuador.

Europa nos recuerda que la gobernanza fluvial se incorpora a la economía, desde Toledo con el Tajo, Paris y el Sena, Roma y el Tiber son además de ser parte de la decoración de la ciudad un elemento indiscutido de la identidad de estas ciudades y sus áreas aledañas son zonas de valor cultural y de desarrollo económico. Así pasamos a América del Norte, en Chicago el rio es el lugar de manifestación de la cultura incluso siendo teñido de verde cuando se celebra la fiesta irlandesa, pero además es el lugar donde se encuentra el desarrollo arquitectónico de la ciudad. En América del Sur en Buenos Aires con puerto madero y el rio de la Plata; en Guayaquil - Ecuador en el rio Guayas se realizó un parque lineal durante un periodo de 17 años consolidando su identidad como una ciudad portuaria y fluvial, sin dejar de nombrar a ManausBrasil y su punta negra, la zona exclusiva de la ciudad. En Colombia son excepcionales por no decir inexistente las situaciones de reubicación en áreas aledañas a los cursos del rio en el 
marco de la planeación territorial.

\section{En transporte}

En materia de transporte Fluvial la Ley 1242 de 2008 establece el Código Nacional de Navegación y Actividades Portuarias Fluviales y dicta otras disposiciones como: "se declara como bien de uso público la franja de terreno que se extiende treinta (30) metros por cada lado del cauce, medidos a partir de la línea en que las aguas alcancen su mayor incremento. La servidumbre legal de uso público en las riberas de las vías fluviales cuya navegación corresponde regular y vigilar a la Nación".

Colombia cuenta con cuatro conjuntos de cuencas principales: las cuencas del Atrato y del Magdalena y las cuencas del Orinoco y el Amazonas. Las primeras fluyen hacia el Mar Caribe y las segundas hacia el Océano Atlántico. Como se puede encontrar en el Plan Maestro Fluvial (MT, 2015 ), los retos que el país tiene respecto al trasporte fluvial son grandes. Existe un vacío referente a la infraestructura (falta de mantenimiento apropiado de instalaciones fluviales como puertos, muelles, falta de conexiones intermodales, etc.), igualmente, se evidencia la falta de respaldo gubernamental al no percibirse una visión a largo plazo de un megaproyecto como este. La utilización estratégica de los ríos como medios de transporte de carga y de pasajeros es bastante limitada en Colombia debido a que se le ha dado prioridad al transporte terrestre y aéreo. La gran problemática es quién es la autoridad que efectivamente cumple con la vigilancia del cuidado de esta franja, pues el Ministerio de transporte no es competente para esto, y solo puede ejercer acciones administrativas por intermedio de la ANI, el INVIAS, CORMAGDALENA o la SUPERTRANSPORTE para las áreas concesionadas, y así las cosas no existen lineamientos intergubernamentales para este fin.

\section{En materia de concesiones portuarias}

El bien de uso público adyacente a la corriente de agua puede ser concesionado para ser utilizado como facilidad portuaria. En materia de concesiones portuarias estas se rigen en general por el Estatuto de Puertos Marítimos o Ley $1^{\text {a }}$ de 1991, que desde su aprobación ha permitido una transformación en términos de eficiencia y desarrollo de la actividad portuaria. Ley $1^{\mathrm{a}}$ definió las facultades respecto de las actividades portuarias, las cuales, serían competencia de la Superintendencia General de Puertos, "entidad creada mediante el mismo Estatuto que tiene funciones específicas en el otorgamiento, modificación, declaratoria de caducidad de las concesiones, control en la reconstrucción de puertos, muelles y embarcaderos, entre otras" (Ley 1, 1991, págs. Arts 26- 27).

La organización del sistema portuario tuvo como objeto propiciar la inversión de origen privado para constituir sociedades portuarias desarrollando su infraestructura y buscando la descentralización mediante la autorización de la Nación a entidades territoriales y descentralizadas para participar en la constitución de las Sociedades Portuarias Regionales. 
Un estudio realizado por el Banco Mundial (Mundial, 2007) sobre la situación actual y perspectivas de los puertos públicos en Colombia muestra que, existe una fragmentación institucional en aproximadamente 5 entidades competentes, hay perdida de especialización dado que la Superintendencia General de Puertos, hoy de transporte (Supertransporte, 2018) controla otros modos de transporte y por último, se evidencia la falta de presencia de esta en los distintos puertos del país. Esto conlleva a la multiplicidad y falta de coordinación en los procesos administrativos del Estado haciendo que la actividad portuaria no obtenga los beneficios esperados. En este sentido es importante recordar que para la cuenca del Magdalena quien la administra es CORMAGDLANA, y para el resto de las cuencas su desarrollo y mantenimiento depende del INVIAS.

\section{En materia pesquera}

Los recursos pesqueros se encuentran definidos en la ley 13 de 1990 que tiene por objeto "regular el manejo integral y la explotación racional de los recursos pesqueros con el fin de asegurar su aprovechamiento sostenido". De tal forma, el Estado se blinda y ejerce gobernanza a las actividades pesqueras.

Al respecto, existen graves vacíos institucionales, por ejemplo, el Ministerio de Agricultura administra los recursos pesqueros y el ministerio de ambiente es el encargado de velar por la protección de las especies. Así, si las especies se encuentran dentro de parques nacionales naturales protegidos por el Ministerio de medio ambiente son recursos para conservar, pero si ese animal sale se vuelve un recurso pesquero (Duarte, 2018).

Tales inconsistencias son consecuencia de la falta de coordinación institucional y de la fragmentación en la que dividieron los ríos y las cuencas. Es de vital importancia la conservación de todo el cauce del rio como un solo ente que preserve todo su sistema ecológico (Duarte, 2018).

\section{En materia minera}

La ley 685 de 2001, tiene como propósito el fomento de la

“exploración técnica y la explotación de los recursos mineros de propiedad estatal y privada; estimular estas actividades en orden a satisfacer los requerimientos de la demanda interna y externa de los mismos y a que su aprovechamiento se realice en forma armónica con los principios y normas de explotación racional de los recursos naturales no renovables y del ambiente, dentro de un concepto integral de desarrollo sostenible y del fortalecimiento económico y social del país". (ley 685 de 2001)

Por esta razón regula las concesiones para la extracción de materiales que son objeto de concesión para su utilización minera, las cuales se basan en principios propios, es de destacar, que son las riveras de los ríos el lugar donde se hace la extracción de los materiales de construcción (Ley 685 2001, art 11), además de tener entre sus preceptos las de área en corrientes de agua (Ley 685 2001, art 64), en extensiones de hasta dos kilómetros y áreas en otros terrenos donde se excluyen las corrientes de agua (Ley 685 2001, art 65). 
Por último, es necesario recordar que, en materia de generación de energía al extender la concesión de la ANLA, se dan los parámetros del concedente para poder realizar el proyecto, pero es claro que es el concesionario el encargado del cuidado de las orillas del cuerpo de agua, no es así en materia de transporte dentro del espejo de agua que seguirá en cabeza del Ministerio de transporte.

\section{Gestión de riesgos}

Con la ley 1523 de 2012 se planteó la política nacional de gestión del riesgo de desastres y se estableció el sistema nacional del mismo. Se planteó la necesidad de aproximarse desde el ordenamiento territorial al conocimiento de los potenciales y debilidades de los territorios y por tanto se hace esencial en la evaluación de cualquier sistema de movilidad y transporte. Además, tiene que ver con la ribera del rio, por esto su importancia incrementa si se tiene en cuenta que todos los organismos estatales deben apoyar y tomar medidas pertinentes que minimicen los riesgos, siendo la zona limitante del rio una de las más expuestas a cambios por la corriente.

La información y los estudios técnicos que da el IDEAM son una determinante para el desarrollo de Ios POT, pero además para un ejercicio preventivo de gestión de riesgos evitando las calamidades que cada año se ven ya sea de inundaciones o sequias. La gobernanza fluvial establece y dirige proyectos que prevengan situaciones de estos tipos dando una mejor distribución de los recursos, pero también evitando el gasto de muchos recursos económicos que provienen de la deficiente planificación sobre la cuenca y el no respeto a la ronda del rio y sus afluentes, sin embargo, no se han logrado alienar parámetros para que zonas de inundación o de potenciales crecientes no sean construidas y en cada época invernal se tiene la misma problemática, lo que permite ver que no es un instrumento de planificación y que la gobernanza en este sentido es precaria.

La no observancia de la prevención, control y cuidado de las rondas de los ríos ha hecho que cada año en temporada de lluvias existan incidentes penosos por falta de planeación, ya sea en Armero, Florencia, El Yopal o Mocoa, y muchos más con consecuencias en pérdidas humanas y daños a infraestructura, sin embargo, solo tiene competencias para la reubicación de personas una vez ocurrida una situación de afectación, sin que sea efectiva una expropiación preventiva.

\section{Implementación en Colombia}

En muchos de los temas existen procedimientos administrativos que se llevan bajo las leyes anteriormente mencionadas, pero por ser de diferentes temas, no relacionados entre sí, pueden existir confusiones en la aplicabilidad de la ley dado que las distintas jurisdicciones no trabajan coordinadamente trayendo como resultado un inadecuado uso de las riberas, especialmente en zonas donde en época de invierno se alcanza la cota más alta de nivel de agua.

\section{El río Magdalena}

En materia de gobernanza fluvial el rio Magdalena es especial por tener un régimen constitucional, y esto le da prioridad, pero al mismo tiempo conduce a grandes dificultades, pues existe una gran cantidad de actores estatales que participan en el proceso teniendo cada uno de los participantes competencias y jurisdicciones que son difícilmente separables. Es decir, en este 
caso la administración del río Magdalena tiene un eje central de importancia, dado a la cantidad de actores que intervienen desde el punto de vista administrativo.

Así la ley 161 de 1994 en el artículo 3 establece la jurisdicción así: “La Corporación Autónoma Regional del Río Grande de la Magdalena Cormagdalena tendrá jurisdicción en el territorio de los municipios ribereños del Río Magdalena". La misma norma habla del ordenamiento de cuenca en los siguientes términos "Cormagdalena estará investida de las facultades necesarias para la coordinación y supervisión del ordenamiento hidrológico y manejo integral del Río Magdalena. La Corporación coordinará, “(...) las actividades de las demás corporaciones autónomas regionales encargadas por la ley de la gestión medio ambiental en la cuenca hidrográfica del Río Magdalena y sus afluentes, en relación con los aspectos que inciden en el comportamiento de la corriente del río, en especial, la reforestación, la contaminación de las aguas y las restricciones artificiales de caudales."

Desde el punto de vista de transporte intervienen el Ministerio de Obras Públicas- Dirección del modo acuático, el Invias, la ANI, la Superintendencia de Puertos y Transportes, CORMAGDALENA y la DIMAR. Desde el punto de vista ambiental son 11 Corporaciones Autónomas Regionales, y en cuanto a las autoridades administrativas son 9 Departamentos, 1 Distrito Especial y en el caso de los municipios solo hasta Barrancabermeja son 54, (72 en total), además de la AUNAP en materia de pesca y la DIAN para el régimen de impuestos y rentas nacionales, esto sin tener en cuenta el nuevo proyecto de Ley de hidrovía del Magdalena.

Para hacer un análisis, es pertinente ver la figura 1. Desde Barrancabermeja hasta la desembocadura existen una cantidad de autoridades, no solo ambientales y administrativas, también de transportes. No se ve coherencia y lineamientos que direccionen el trabajo de todos hacia un mismo camino creando falencias en su efectividad. La misma situación se ve en los demás cursos de agua en el país con la diferencia de que no actúa Cormagdalena, siendo más complejo por ser la ruta estratégica para transporte de hidrocarburos.

Figura 1. Distribución de jurisdicción de las autoridades regionales

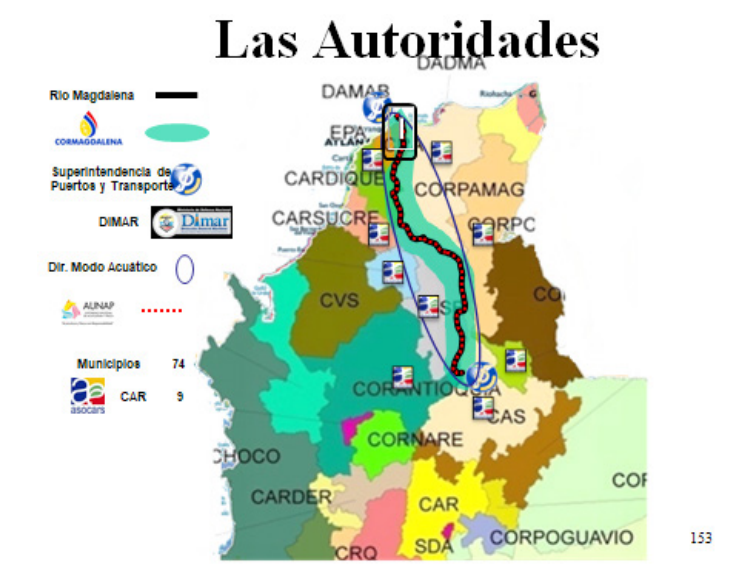

Fuente: Elaboración propia 
Corporaciones Autónomas Regionales y de Desarrollo Sostenible y su jurisdicción

La jurisdicción de las Corporaciones autónomas tiene un amplio marco normativo como la Ley 3 de 1961 y modificada por las Leyes 62 de 1984 y 99 de 1993, entre otras y en ellas además de sus funciones, se establecen las jurisdicciones a las que cada una de ellas pertenece. En el mapa se puede observar cómo estas autoridades no corresponden necesariamente a un ente territorial, y que muchas llevan implícito en su nombre un rio o cuerpo de agua que los identifica. (Ver figura 2).

Figura 2. Corporaciones autónomas regionales y de desarrollo sostenible

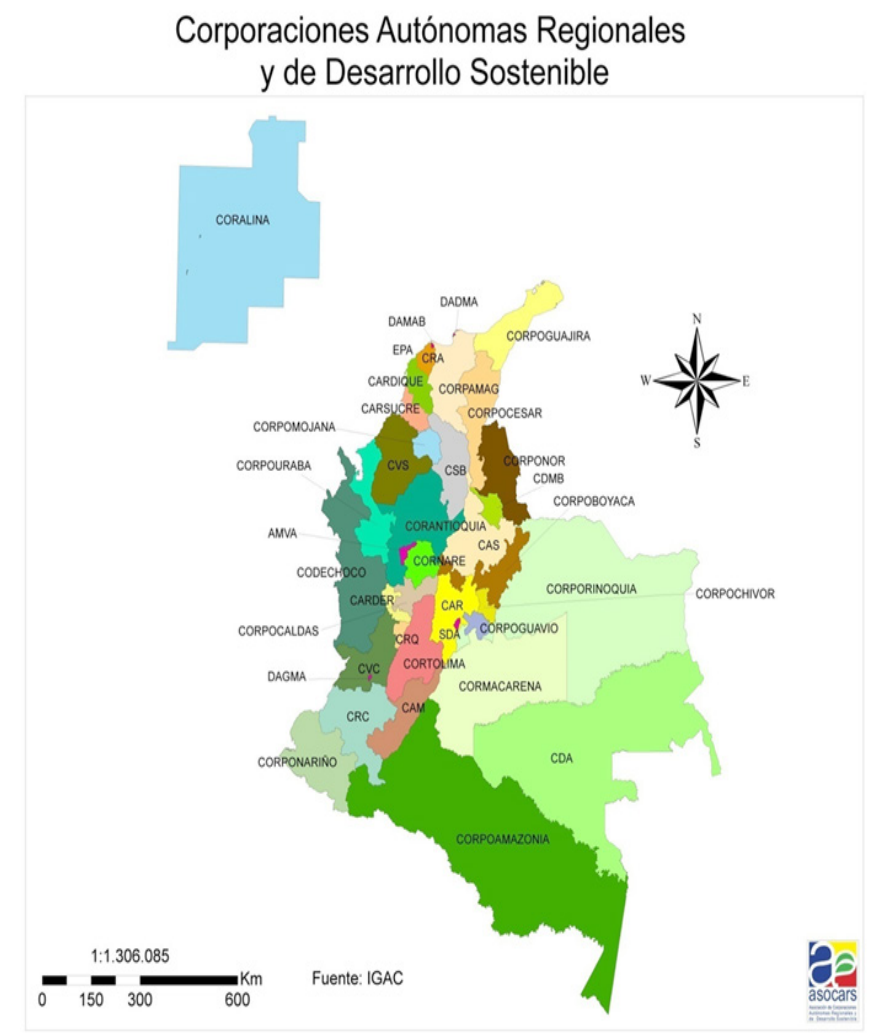

Fuente: https://es.slideshare.net/alexa842003/asocars

Ahora bien al observar los POMCAS establecidos en los planes de ordenación y manejo de cuencas, se observan 5 grandes macro cuencas de donde se desprenden 57 cuencas y 309 microcuencas, de acuerdo a sus funciones, las autoridades ambientales no deberían romper los sistemas naturales por su jurisdicción, precisamente por ser las garantes del manejo de los recursos hídricos, si esto pasa en el ejercicio administrativo se dificulta más el control de los bienes de usos públicos, como se observa en la figura 3. 
Figura 3. Estructura de planificación, ordenación y manejo de cuencas hidrográficas y acuíferos

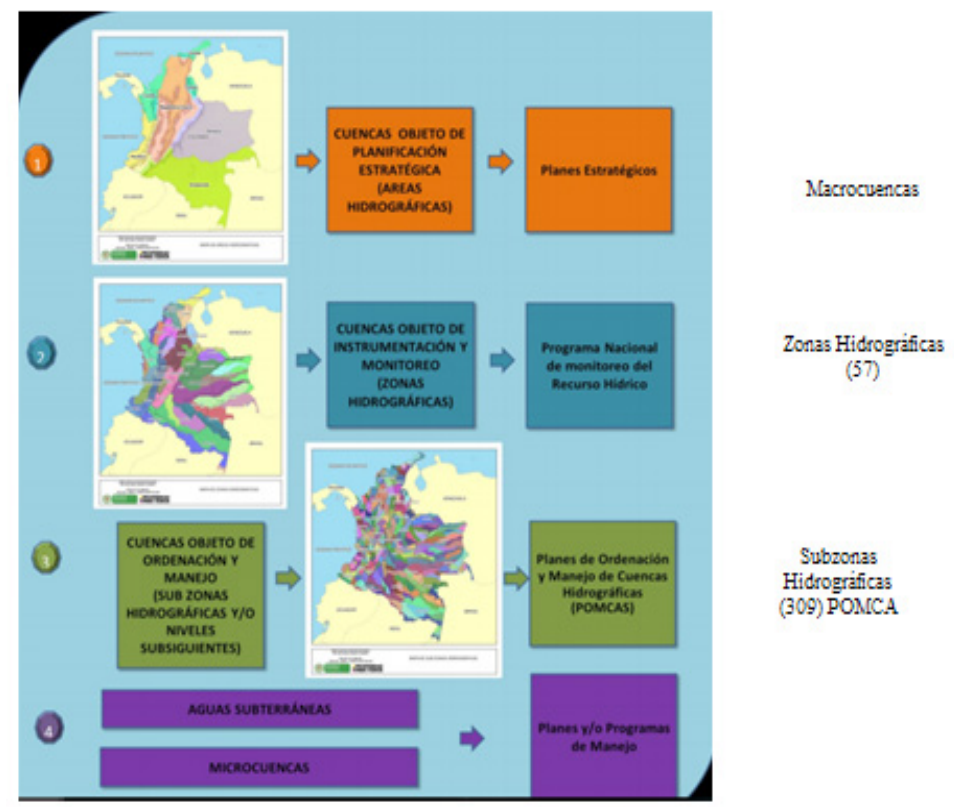

Fuente: Ministerio de Ambiente, Vivienda y Desarrollo Territorial, 2010. P. 19

En este sentido, tal como lo ha indicado Zamudio (2012), la gobernabilidad del agua hace parte del gran entramado del que se componen los sistemas políticos. Los factores políticos, ambientales, económicos, sociales y administrativos deben unirse para regular el desarrollo y la gestión de los recursos hídricos, en todo caso la segmentación administrativa en lo ambiental y desde la jurisdicción de las corporaciones autónomas no es coherente con la división natural que establecen las cuencas, en este caso el hombre hace prevalecer y dividir los sistemas.

\section{Casos en ciudades que miran el río en Colombia}

\section{Medellín}

La gobernanza tomada por el liderazgo de las ciudades ribereñas está transformando la cultura y la forma de vida en las ciudades. Brinda oportunidades turísticas a propios y foráneos y permite la realización de intervenciones urbanas en el entorno inmediato (Marriaga, 2010), dándole incluso identidad a sus habitantes.

El megaproyecto parques del rio Medellín hace parte del POT de Medellín donde se proyecta la reorganización de la ciudad a lo largo del rio. Se inició en el año 2015 y tiene el fin de aumentar la conectividad a través de la instalación de puentes que comunican los dos costados del rio que sirven a su vez, como principal corredor ecológico de la ciudad y de la región. 


\section{Bogotá}

En el caso de Bogotá en el año 2017 se propuso la creación de un gran parque lineal de más de 1000 hectáreas en el cual converjan espacios recreativos y ambientales. En palabras del exalcalde Enrique Peñalosa, "el rio será el eje de la vida en la ciudad, la columna vertebral de la integración social”. (Giraldo, 2018)

Gracias a esta decisión Bogotá y el país dejan de darle la espalda al río, que dejará de ser la cloaca en que se había convertido, para transformarse en el eje articulador del desarrollo de la ciudad y de la región.

\section{Montería}

El Parque Lineal la Ronda del Sinú, integra objetivos ambientales como la reducción de emisiones de gases y la conservación ambiental con escenarios de recreación, deporte, cultura y educación ambiental. El proyecto genera a su vez actividades económicas que benefician el comercio de alimentos para el aprovechamiento de ciudadanos de estratos medios y bajos. (Aguilar, 2013)

\section{Barranquilla}

Barranquilla y su área metropolitana cuentan con un gran equipamiento urbano reflejado en puertos marítimos, fluviales y aéreos. La mala planificación ha llevado a que varios sectores sean relegados y subutilizados (Marriaga, 2010), a pesar de ello, el Malecón y la vía del río acompañado de una infraestructura recreativa, permiten que la ciudad mire el rio desarrollándose actividades sociales y culturales en la ribera.

El modelo de ordenamiento territorial busca aprovechar los máximos beneficios que brindan el rio, el mar y los demás ecosistemas para conseguir la integración en aspectos económicos, sociales y funcionales. De igual forma, buscan asegurar la conectividad regional, nacional e internacional articulando el sistema vial metropolitano con la red vial regional y nacional, y estableciendo una comunicación multimodal que facilite el acceso de la metrópoli a los mercados nacionales e internacionales (Berdugo, 2018).

\section{Aplicación de la norma}

En este aspecto se han dado importantes resultados desde el punto de vista del resarcimiento de derechos específicamente para las cuencas o ríos. Así por ejemplo, dentro del expediente núm. AP-25000-23-27-000-2001-90479-01, el Consejo de Estado se manifiesta en el caso del rio Bogotá respecto al alto grado de contaminación debido a vertimientos de aguas residuales domésticas e industriales, malas prácticas agropecuarias e inadecuado manejo de basuras por parte de los habitantes e industrias aledañas, además de la omisión de las autoridades frente a estas situaciones por más de treinta años (IDEAM, 2020), buscando su recuperación y dando una serie de órdenes a las diferentes entidades estatales (Nacionales, Departamentales, Distritales y municipales) para que se logre su descontaminación buscando proteger derechos ambientales de calidad del agua, ecológicos de defensa de patrimonio público, cultural, entre otros. 
La Corte Constitucional mediante la sentencia T-622 de 2016 reconoce al río Atrato como sujeto de derechos con miras a garantizar su conservación y protección, en su decisión ordena al Gobierno Nacional elegir un representante legal de los derechos del río, y mediante el decreto 1148 de 2017 el Presidente de la República designó al Ministerio de Ambiente y Desarrollo Sostenible como representante legal, además decide la conformación de una comisión de guardianes comprendida por un representante del Gobierno Nacional y uno de las comunidades, además prevé la construcción de diferentes planes de acción en conjunto con las comunidades para resolver la crisis humanitaria, social y ambiental que presenta el río Atrato, sus afluentes y sus comunidades.

El diseño y construcción de estos planes de acción debe hacerse de manera conjunta con otras entidades que ordena la Corte dentro de la Sentencia, como Presidencia de la República, el Ministerio de Defensa, Salud, Agricultura, Hacienda, Minas, Ambiente, DNP, DPS, Corpourabá, Codechocó, las gobernaciones y alcaldías que hacen parte de la cuenca, y las demás entidades que tengan conocimiento científico y técnico en relación con el proceso de recuperación del río (Minambiente, 2020).

Por su parte el tribunal superior de Medellín en sentencia reconoce al río Cauca como sujeto de derechos de especial protección, lo que conlleva a velar por la Inter institucionalidad para su protección, conservación, mantenimiento y restauración, y que las generaciones futuras tienen derechos fundamentales como el gozar de la naturaleza, el agua, seguridad alimentaria y un medio ambiente sano (uniminuto, 2019), de todo esto se desprende que el gobierno en general debe implantar medidas que protejan los cursos de agua y sus cuencas, y que quienes habitan en ellas serán sujetos que se involucren en la gobernanza.

"Estas decisiones les ha dado eficacia jurídica a estas decisiones, colectivas, participativas, consensuadas, en los asuntos de los regantes. En nuestro contexto latinoamericano, varios países reconocen formalmente las formas comunitarias de gestión" (OAS, 2020, pág. 20), entre los cuales se encuentra Colombia.

\section{Hallazgos y análisis}

La Gobernanza fluvial en Colombia tiene zonas grises jurisdiccionales debido a que no se tiene un control efectivo de las áreas fluviales y la coexistencia de muchas autoridades con acciones administrativas efectivas que, aunado a la no recordación de la historia con incidentes catastróficos, no han producido efectos en la administración del recurso.

No se conoce una acción administrativa de ninguna autoridad que busque proteger el área de los 30 metros como bien de uso público.

En el contexto nacional se ven ciudades como Bogotá, Barranquilla, Medellín y Montería, las cuales tienen dentro de sus planes de desarrollo un nuevo enfoque que integra los proyectos urbanísticos con el medio ambiente y el desarrollo sostenible dando un ejemplo de clara gobernanza en lo urbano y aprovechamiento de la riqueza natural limitando el desgobierno fluvial en estas zonas. 
Dentro de las funciones administrativas no es claro en la ley 1242 de 2008 si el funcionario público tiene competencia para ejercer la función del cuidado de estas áreas, sin embargo, la autoridad ambiental tampoco efectúa los procedimientos correspondientes necesarios para la recuperación y el cuidado del bien de uso público.

Existen una serie de normativas importantes que pueden ayudar a dar prelación y cohesión institucional y esto es contundente en el caso de las ciudades que miran el rio.

La gestión de riesgos se queda corta, pues debería poder definir por medio de estudios y planeación áreas de anegación las cuales no pueden ser utilizadas para zonas de habitación y restricción para cultivos, esto acompañado de la información dada por el IDEAM.

La jurisdicción de las autoridades regionales de medio ambiente no fue concebida de acuerdo con el trazado de las cuencas y microcuencas, lo que dificulta una efectiva gestión rompiendo con el sistema natural y aunque no se estudian la cuenca como tal, los temas piscícolas o el uso del agua como fuente energética, todas estas acciones vuelven más complejo el manejo del recurso, más cuando está liderado por diferentes organismos que no tienen coordinación, ya que ni siquiera pertenecen al mismo ente general del ejecutivo, llámese transporte, agricultura o ambiente.

\section{Conclusiones}

La investigación revela de forma contúndete que la interferencia de distintos y tan variados actores tanto como del sector público como del privado hace difusa la consolidación de un proyecto claro de gobernanza fluvial al existir multiplicidad de tareas y escaso control real sobre el tema.

La deficiente sinergia legislativa crea zonas grises para la gobernanza al existir una serie de normas de carácter legal de diferente índole temática y sin cohesión institucional no son suficientes los esfuerzos legales y se requiere de un ente cohesionador que permita hacer todas las actividades pero que a su vez límite hasta donde cada autoridad puede llegar y concretar por medio de la aplicación del código administrativo un responsable para definir estos casos.

Al no tenerse en cuenta en la administración ambiental el sistema de cuencas y microcuencas se puede estar cercenando el sistema como un todo y no permite un control eficiente de los recursos hídricos ni de sus riberas.

La problemática de no tener un doliente efectivo permite que no exista un control y por lo tanto, la no gobernanza de grandes áreas, dando lugar a que se presenten casos como la destrucción de cursos de agua por explotación de minería ilegal.

Al existir zonas grises no es claro cuál sea la autoridad ambiental, la responsable de mantener este bien de usos públicos perenne para los habitantes de Colombia, entre más autoridades existan menos ejercicio de control efectivo. Además de que requiere de una infraestructura importante que salvaguarde los bienes de usos públicos. 
No existe un proceso administrativo de ninguna índole para la restitución al estado de estos bienes de uso público, a diferencia de lo que ocurre en las áreas marino-costeras.

La gestión de riesgos podría tomar medidas que permitan la recuperación de estos bienes de uso público.

La unión de esfuerzos alineados en un objetivo como la recuperación de las áreas de uso público fluvial permite que se afinen las reglas para su uso.

\section{Referencias}

Aguilar, E. (2013). http://www.institut-gouvernance.org/es/experienca/fiche-experienca-33. html. Obtenido de http://www.institut-gouvernance.org/es/experienca/ fiche-experienca-33.html

Berdugo, J. (15 de 2 de 2018). https://ww2.camacolcundinamarca.co/documentos/ presentaciones/HacialaGranMetropoliCaribe-Jaime-Berdugo.pdf. Obtenido de https:// ww2.camacolcundinamarca.co/documentos/presentaciones/HacialaGranMetropoliCaribe-Jaime-Berdugo.pdf

DNP. (2019). Recuperadohttps://proyectostipo.dnp.gov.co/normatividad/BasesPND2014-2018 Bases del Plan Nacional de Desarrollo 2018 -2022: un pacto por Colombia, un pacto por la equidad. Departamento Nacional de Planeación. . Obtenido de https:// proyectostipo.dnp.gov.co/normatividad/BasesPND2018-2022.pdf

Duarte, L. O. (18 de 01 de 2018). Obtenido de https://sostenibilidad.semana.com/ medio-ambiente/articulo/asi-esta-la-pesca-artesanal-en-colombia/39351: https:// sostenibilidad.semana.com

Giraldo, M. (15 de 2 de 2018). http://www.bogota.gov.co/temas-de-ciudad/obras-y-desarroIlo-urbano/descontaminacion-del-rio-bogota-alcaldia-penalosa-construira-planta-canoas. Obtenido de http://www.bogota.gov.co/temas-de-ciudad/obras-y-desarrollo-urbano/descontaminacion-del-rio-bogota-alcaldia-penalosa-construira-planta-canoas

Hall, P. R. (2003). https://www.cepal.org/samtac/noticias/documentosdetrabajo/0/23420/ GWP00504.pdf. Obtenido de https://www.cepal.org

Iza, A. O. (Alejandro O. Iza y Marta B. Rovere (Editores) (2006). Gobernanza del agua en América del Sur: dimensión ambiental. UICN, Gland, Suiza y Cambridge, Reino Unido. de 2006). Gobernaza del agua en America del Sur: dimensión ambiental. https://www. iucn.org/es/content/gobernanza-del-agua-en-america-del-sur-dimension-ambiental-0. Gland y Cambridge, Suiza, Reino Unido: UICN CAF.

Ley1. (1991). Ley. Bogota: Congreso Republica.

Marriaga, A. D. (2010). https://issuu.com/cideu/docs/alfonso_segundo_de_la_cruz_-metro_ rio_entrega_fin. Obtenido de cideu: https://issuu.com/cideu/docs/alfonso_segundo_ de_la_cruz_-metro_rio_entrega_fin 
Minambiente. (2 de 4 de 2020). Obtenido de https://www.minambiente.gov.co/index.php/ component/content/article/3573-sentencia-t-622-de-2016-rio-atrato-como-sujeto-dederechos

Minas, M. d. (2001). Codigo de Minas. Bogota: Congreso de Republica.

MT, D. . (2015 ). Recuperado de: https://onl.dnp.gov.co/es/Publicaciones/Paginas/ Plan-Maestro-Fluvial.aspx. Obtenido de ARCADIS Nederland: https://onl.dnp.gov.co/

Mundial, B. (1 de 04 de 2007). http://documents1.worldbank.org/curated/ en/782761468027627013/pdf/506810WP0SPANI10Box342005B01PUBLIC1.pdf. Obtenido de Construir los fundamentos para la competitividad. Situación Actual y Perspectivas de los Puertos Públicos en Colombia: http://documents1.worldbank.org

oas.org. (04 de 3 de 2020). http://www.oas.org/en/sedi/dsd/IWRM/Past\%20Events/ D7/6\%20WWF-GOBERNANZA\%20Final.pdf. Obtenido de HACIA UNA BUENA GOBERNANZA DE LOS RECURSOS HIDRICOS/ Judith Domínguez Serrano: http://www. oas.org

Política, C. (1991). Bogota.

Supertransporte. (2018). Decreto 2409. Bogota: Congreso.

uniminuto. (20 de 06 de 2019). Obtenido de https://www.uniminutoradio.com.co/fallo-historico-reconocen-al-rio-cauca-como-sujeto-de-derechos/

www.ideam.gov.co. (2 de 04 de 2020). Obtenido de http://www.ideam.gov.co/web/ocga/ sentencia

Zamudio Rodríguez, C (2012). Gobernabilidad sobre el recurso hídrico en Colombia: Entre avances y retos. Gestión y Ambiente, Vol. 15, No. 3, p.99-112, ISSN: 2357-5907. Obtenido de: https://revistas.unal.edu.co/index.php/gestion/article/view/36284 